\title{
Dynamic Behavior and Exact Expressions of Traveling Wave Solutions for a class of nonlinear partial differential equations
}

\author{
Ruo Feng Zhang \\ School of Mathematics and Statistics, Tianshui Normal University, Gansu \\ Tianshui 741001, China \\ zrfmath@163.com
}

\begin{abstract}
The partial differential equation generated by one of the Jaulent-Miodek hierarchy models is completely integrable. Applying bifurcation method, some new exact traveling wave solutions of it such as kink wave solutions and unbounded wave solutions are obtained, which corresponding to the solitary wave solutions of its traveling wave system.
\end{abstract}

Keywords: Bifurcation, Solitary wave solution, Unbounded wave solutions.

\section{Introduction}

In this paper, we discuss system

$$
u_{x t}+\frac{1}{4} u_{x x x x}-\frac{3}{2} u_{x}^{2} u_{x x}+\frac{3}{16} u_{y y}+\frac{3}{4} u_{x x} u_{y}-\alpha u_{z z}=0,
$$

where $\alpha$ is constant ${ }^{[1]}$. The traveling wave system of (1.1) is derived ${ }^{[5]}$. Wazwaz ${ }^{[1]}$ has obtained by Hirota bilinear method multiple soliton solutions which were formally derived. In this paper, we research the travel wave solutions of (1.1) by bifucation method of dynamical systems ${ }^{[3,5]}$. 


$$
\begin{aligned}
& \frac{d \varphi}{d \xi}=y, \\
& \frac{d y}{d \xi}=-\frac{\left(3 r^{2}-16 k c-16 \alpha\right)}{4 k^{4}} \varphi-\frac{3 r}{2 k^{2}} \varphi^{2}+2 \varphi^{3}
\end{aligned}
$$

where $u_{\xi}=\varphi, \quad \xi=k x+r y+z-c t$.

The system(1.2) has solitary wave solutions, which have been found in [1,2] completely. In this paper, we will research a new class of wave solutions of(1.1) which correspond to solitary wave solutions of(1.2). This paper is organized as follows. In section 2, all the possible travel wave solutions of the system(1.1) are given, which correspond to solitary wave solutions of the system(1.2). Finally, the physical significance of this new traveling wave solutions of(1.1) are given.

\section{Exact traveling wave Solutions of the system (1.1)}

\section{Lemma 1}

the system (1.2) has valley and peak type solitary wave solutions as follows (see Fig4(1,2,3,4)):

(1) If $\quad(a, b) \in I I \quad$ then $(k, r, c, \alpha) \in\left\{(k, r, c, \alpha) \mid k c+\alpha \geq 0,-\frac{4 \sqrt{3(k c+\alpha)}}{3}<r<-2 \sqrt{(k c+\alpha)}\right\}$ , (1.2) has a valley type solitary wave solution ${ }^{[5]}$

$$
\begin{aligned}
\varphi_{1}(\xi) & =\frac{2 M_{1} m_{1}}{\left(M_{1}-m_{1}\right) \cosh \left(\sqrt{m_{1} M_{1}} \xi\right)+m_{1}+M_{1}}, \\
& =-\frac{18 k^{2} a}{k^{2} \sqrt{\Delta_{1}} \cosh (\sqrt{a} \xi)-9 r} .
\end{aligned}
$$


where $\quad m_{1}=\frac{r-36 \sqrt{\Delta_{1}} k^{2}}{2 k^{2}}<M_{1}=\frac{r+36 \sqrt{\Delta_{1}} k^{2}}{2 k^{2}}<0$

$\Delta_{1}=\frac{324\left(r^{2}-4 k c-4 \alpha\right)}{k^{4}}$.

(2) If $(a, b) \in I I I$, then $(k, r, c, \alpha) \in\{(k, r, c, \alpha) \mid k c+\alpha<0, r<0\}$ 或 者

$\left\{(k, r, c, \alpha) \mid k c+\alpha \geq 0, r<-\frac{4 \sqrt{3(k c+\alpha)}}{3}\right\},(1.2)$ has a valley type solitary wave solution ${ }^{[5]}$

$$
\varphi_{2}(\xi)=\varphi_{s 1}-\frac{2 \tilde{a}_{1}}{\sqrt{\tilde{b}_{1}^{2}+4 \tilde{a}_{1}} \cosh \left(\sqrt{\tilde{a}_{1}} \xi\right)-\tilde{b}_{1}},
$$

where $\tilde{a}_{1}=\left(M_{1}-\varphi_{s 1}\right)\left(m_{1}-\varphi_{1}\right), \tilde{b}_{1}=\left(2 \varphi_{s 1}-M_{1}-m_{1}\right)$.

(3) If $\quad(a, b) \in V I \quad$ then $(k, r, c, \alpha) \in(k, r, c, \alpha) \mid k c+\alpha \geq 0, \frac{8 \sqrt{66(k c+\alpha)}}{33}<r<2 \sqrt{(k c+\alpha)}$, We can obtain the third valley type solitary wave solution $\varphi_{6}(\xi)$ of (1.2) $\varphi_{3}(\xi)$ has the same expression as $\varphi_{2}(\xi)^{[5]}$.

(4) If $\quad(a, b) \in V \quad$ then $(k, r, c, \alpha) \in\left\{(k, r, c, \alpha) \mid k c+\alpha \geq 0,2 \sqrt{(k c+\alpha)}<r<\frac{4 \sqrt{3(k c+\alpha)}}{3}\right\}$,

(1.2) has a peak type solitary wave solution ${ }^{[5]}$

$$
\begin{aligned}
\varphi_{4}(\xi) & =\frac{2 M_{2} m_{2}}{\left(M_{2}-m_{2}\right) \cosh \left(\sqrt{m_{2} M_{2}} \xi\right)+m_{2}+M_{2}}, \\
& =\frac{18 k^{2} a}{k^{2} \sqrt{\Delta_{1}} \cosh (\sqrt{a} \xi)+9 r},
\end{aligned}
$$


where $0<m_{2}=\frac{r-36 \sqrt{\Delta_{1}} k^{2}}{2 k^{2}}<M_{2}=\frac{r+36 \sqrt{\Delta_{1}} k^{2}}{2 k^{2}}$.

(5)

$$
\text { If } \quad(a, b) \in I
$$

then

$(k, r, c, \alpha) \in\left\{(k, r, c, \alpha) \mid k c+\alpha \geq 0,-2 \sqrt{(k c+\alpha)}<r<-\frac{8 \sqrt{66(k c+\alpha)}}{33}\right\}$

, (1.2) has a peak type solitary wave solution ${ }^{[5]}$,

$$
\varphi_{5}(\xi)=\varphi_{s 2}+\frac{2 \tilde{a}_{2}}{\sqrt{\tilde{b}_{2}^{2}+4 \tilde{a}_{2}} \cosh \left(\sqrt{\tilde{a}_{2}} \xi\right)+\tilde{b}_{2}},
$$

where $\tilde{a}_{2}=\left(M_{2}-\varphi_{s 2}\right)\left(m_{2}-\varphi_{s 2}\right), \tilde{b}_{2}=\left(2 \varphi_{s 2}-M_{2}-m_{2}\right)$.

(6) If $(a, b) \in I V$, then $(k, r, c, \alpha) \in\{(k, r, c, \alpha) \mid k c+\alpha<0, r>0\}$ or $\left\{(k, r, c, \alpha) \mid k c+\alpha \geq 0, r>\frac{4 \sqrt{3(k c+\alpha)}}{3}\right\}$. We can obtain the peak type solitary wave solution $\varphi_{6}(\xi)$ has the same expression as $\varphi_{5}(\xi)^{[5]}$.

\section{Theorem 1}

From lemma1, six exact traveling wave solutions (see Fig1(4,5,6,10,11,12)) of (1.1) corresponding to the solitary wave solutions of (1.2) are obtained as follows:

$$
\begin{aligned}
& u_{1}(\xi)=\int-\frac{18 k^{2} a}{k^{2} \sqrt{\Delta_{1}} \cosh (\sqrt{a} \xi)-9 r} d \xi \\
& =-\frac{36 k^{2} \sqrt{a}}{\sqrt{k^{4} \Delta_{1}-81 r^{2}}} \arctan \left(\frac{\left(k^{2} \sqrt{\Delta_{1}}+9 r\right) \tanh \left(\frac{\sqrt{a}}{2} \xi\right)}{\sqrt{k^{4} \Delta_{1}-81 r^{2}}}\right) . \\
& u_{2}(\xi)=\varphi_{s 1} \xi-\int \frac{2 \tilde{a}_{1}}{\sqrt{\tilde{b}_{1}^{2}+4 \tilde{a}_{1}} \cosh \left(\sqrt{\tilde{a}_{1}} \xi\right)-\tilde{b}_{1}} d \xi
\end{aligned}
$$




$$
=\varphi_{s 1} \xi-2 \arctan \left(\frac{\left(\sqrt{\tilde{b}_{1}^{2}+4 \tilde{a}_{1}}+\tilde{b}_{1}\right) \tanh \left(\frac{\sqrt{\tilde{a}_{1}}}{2} \xi\right)}{2 \sqrt{\tilde{a}_{1}}}\right),
$$

$$
\begin{aligned}
& u_{4}(\xi)=\frac{18 k^{2} a}{k^{2} \sqrt{\Delta_{1}} \cosh (\sqrt{a} \xi)+9 r} \\
& =\frac{36 k^{2} \sqrt{a}}{\sqrt{k^{4} \Delta_{1}-81 r^{2}}} \arctan \left(\frac{\left(k^{2} \sqrt{\Delta_{1}}-9 r\right) \tanh \left(\frac{\sqrt{a}}{2} \xi\right)}{\sqrt{k^{4} \Delta_{1}-81 r^{2}}}\right),
\end{aligned}
$$

$$
\begin{aligned}
& u_{5}(\xi)=\varphi_{s 2}+\frac{2 \tilde{a}_{2}}{\sqrt{\tilde{b}_{2}^{2}+4 \tilde{a}_{2}} \cosh \left(\sqrt{\tilde{a}_{2}} \xi\right)+\tilde{b}_{2}} \\
& =\varphi_{s 2} \xi+2 \arctan \left(\frac{\left(\sqrt{\tilde{b}_{2}^{2}+4 \tilde{a}_{2}}-\tilde{b}_{2}\right) \tanh \left(\frac{\sqrt{\tilde{a}_{2}}}{2} \xi\right)}{2 \sqrt{\tilde{a}_{2}}}\right),
\end{aligned}
$$

where $u_{1}(\xi)$ is kink wave solutions (see Fig1(5)) of (1.1), $u_{4}(\xi)$ is anti-kink wave solutions (see Fig1(7)) of (1.1), $u_{3}(\xi)$ which has the same expression as $u_{2}(\xi), u_{6}(\xi)$ which has the same expression as $u_{5}(\xi)$, are exact unbounded traveling wave solutions (see Fig1(2,5)) of $(1.1)$.

Proof :By lemma1 and $u_{\xi}=\varphi$, theorem 1 are easily proved. 


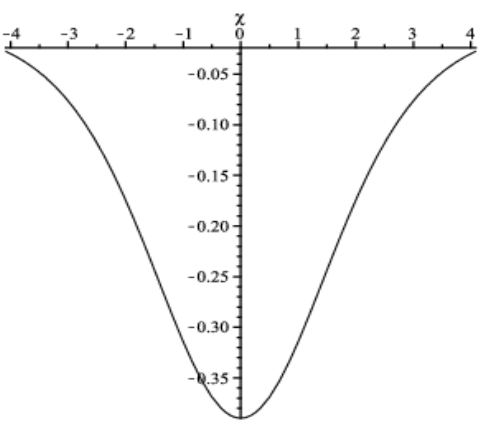

(1) $\varphi_{1}$

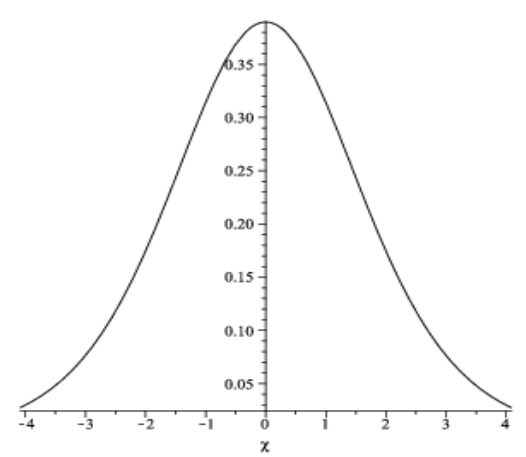

(3) $\varphi_{4}$

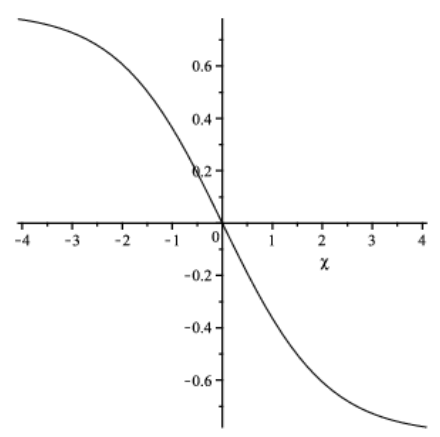

(5) $u_{1}$

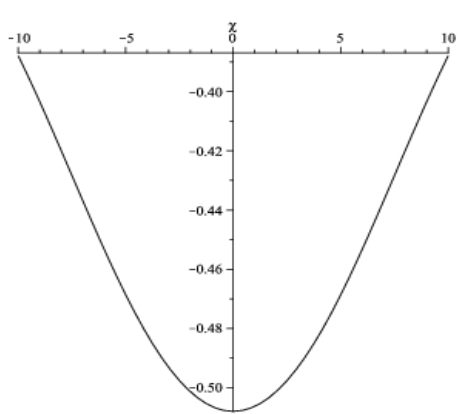

(2) $\varphi_{2}$

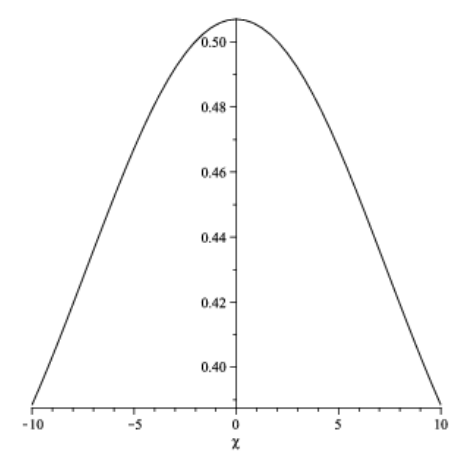

(4) $\varphi_{5}$

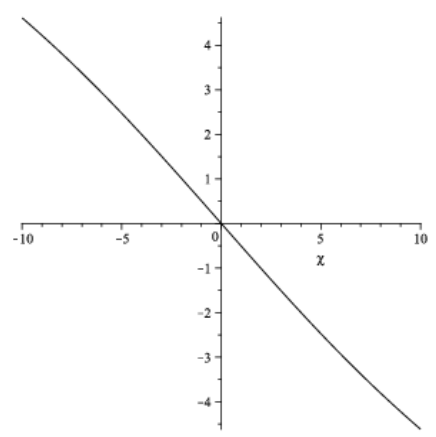

(6) $u_{2}$ 


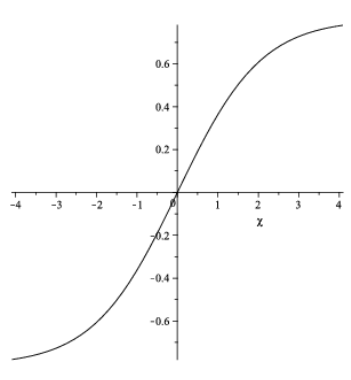

(7) $u_{4}$

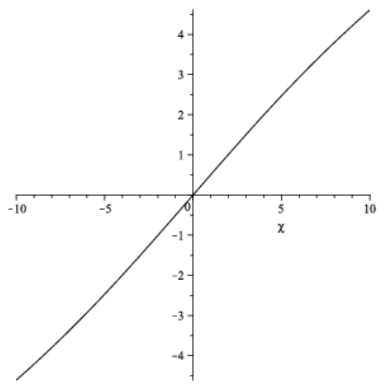

(8) $u_{5}$

Fig1: The kink wave, unbounded wave, anti-kink wave solutions of the system (1.1) corresponding to the solitary wave solutions of the system (1.2).

\section{Conclusions}

In this paper, a class of traveling solutions of (1.1) which are integration of all solitary wave solutions are obtained. These solution are not solitary wave solutions but kink wave solutions, anti-kink wave solutions, unbounded wave solutions. Our results are significant to analyze the integrate of traveling wave solutions.

\section{References}

[1] A. M. Wazwaz, Multiple soliton solutions for some (3+1)-dimensional nonlinear models generated by the Jaulent-Miodek hierarchy, Appl. Math. Lett. 21 (2012), no. 25, 1936--1940.

[2] A. M.Wazwaz, Multiple kink solutions and multiple singular kink solutions for $(2+1)$-dimensional nonlinear models generated by the JaulentCMiodek hierarchy, Phys. Lett.A. 21 (2009), no. 373, 1844-1846.

[3] Li, J. B. Dai, H. H, On the Study of Singular Nonlinear Traveling Wave 
Equations:Dynamical System Approach, Science Press, Beijing, 2007.

[4] J. B. Li and G. R. Chen, Exact Traveling wave solutions and their bifurcations for the Kudryashov-Sinelshchikov equation, Internat. J. Bifur. Chaos Appl. Sci.

Engrg.12 (2012), no. 22(5), 1250118-1-19.

[5] Y. P. Ran and J. Li, Bifurcation Method to Analysis of Traveling Wave

Solutions for $(3+1)$-Dimensional Nonlinear Models Generated by the

Jaulent-Miodek Hierarchy,The Bulletin of the Iranian Mathematical Society.

(2014), in press.

[6] R. Hirota, The Direct Method in Soliton Theory, Cambridge Univ. Press, Cambridge,2004. 